\title{
Connections between jet formation and multiwavelength spectral evolution in black hole transients
}

\author{
Emrah Kalemci ${ }^{1}$, Yoon-Young Chun ${ }^{1}$, Tolga Dinçer ${ }^{1}$, Michelle \\ Buxton $^{2}$, John A. Tomsick ${ }^{3}$, Stephane Corbel ${ }^{4}$ and Philip Kaaret ${ }^{5}$ \\ ${ }^{1}$ Sabanci University, Tuzla \\ İstanbul, Turkey \\ email: ekalemci@sabanciuniv.edu \\ ${ }^{2}$ Department of Astronomy, Yale University, P.O. Box 208101 \\ New Haven, CT 06520-8101, USA \\ ${ }^{3}$ Space Sciences Laboratory, University of California, Berkeley \\ 7 Gauss Way, Berkeley, CA 94720-7450, USA \\ ${ }^{4}$ Laboratoire Astrophysique des Interactions Multi-echelles (UMR 7158) \\ CEA/DSM-CNRS-Universite Paris Diderot \\ CEA Saclay, F-91191 Gif sur Yvette, France \\ ${ }^{5}$ Department of Physics and Astronomy, University of Iowa \\ Van Allen Hall, Iowa City, IA 52242, USA
}

\begin{abstract}
Multiwavelength observations are the key to understand conditions of jet formation in Galactic black hole transient (GBHT) systems. By studying radio and optical-infrared evolution of such systems during outburst decays, the compact jet formation can be traced. Comparing this with X-ray spectral and timing evolution we can obtain physical and geometrical conditions for jet formation, and study the contribution of jets to X-ray emission.

In this work, first X-ray evolution - jet relation for XTE J1752-223 will be discussed. This source had very good coverage in X-rays, optical, infrared and radio. A long exposure with INTEGRAL also allowed us to study gamma-ray behavior after the jet turns on. We will also show results from the analysis of data from GX 339-4 in the hard state with SUZAKU at low flux levels. The fits to iron line fluorescence emission show that the inner disk radius increases by a factor of $>27$ with respect to radii in bright states. This result, along with other disk radius measurements in the hard state will be discussed within the context of conditions for launching and sustaining jets.
\end{abstract}

Keywords. accretion, black hole physics, line: profiles

\section{Introduction}

Hard state of GBHTs is of great interest because emission from all fundamental accretion components are present: the accretion disk, the corona and the jet. In this state, there is also rich timing information as evident from QPOs and broad band timing features in the power spectra. Our group is especially interested in the hard state during the decays of outbursts, as the decays not only involve state transitions in temporal and spectral properties, but the appearance of jet signatures in the radio and near infrared (NIR) also occurs during decays.

Thanks to intense monitoring campaigns in X-rays with $R X T E$ accompanied by radio (especially with ATCA and VLA) and NIR (with SMARTS) of GBHTs, we were able to get a general picture of evolution during decays: A fast timing transition (sudden increase in rms amplitude of variability accompanied by an increase in power-law flux) is followed 
by a hardening a few days to weeks later. Close to the end of hardening, jet signatures are observed in NIR and radio. In some cases, a softening is observed at very low flux levels (see Kalemci, et al. 2006a for detailed description). Moreover, observations with HEXTE on $R X T E$ indicate disappearance of breaks in the high energy spectra of GBHTs after jet turn on (Kalemci, et al. 2005 and 2006b). We continue to monitor GBHTs during decays to improve the overall picture, while extending our studies to the evolution of iron line features and timing at low flux levels.

\section{Multiwavelength evolution of XTE J1752-223}

GBHT XTE J1752-223 was discovered by RXTE in October 2009 (Markwardt, et al. 2009). The outburst evolution was monitored intensely by RXTE (Shaposhnikov, et al. 2010), MAXI (Nakahira, et al. 2010) and SWIFT (Curran, et al. 2010). Its X-ray properties are consistent with being a black hole (Munoz Darias, et al. 2010).

The source was also monitored in radio (Brocksopp, et al. 2009) and observed with EVN and VLBA in radio (Yang, et al. 2010). Finally, it is monitored with the Faulkes Observatory in optical (http://staff.science.uva.nl/ davidr/faulkes/xtej1752.html) and also with the SMARTS CTIO 1.3m Telescope in optical (I band) and in NIR (Buxton, et al. 2009).

We concentrated on the decay of outburst and characterized the X-ray spectral evolution using the PCA on $R X T E$. We fit the X-ray spectrum with absorption, disk blackbody,

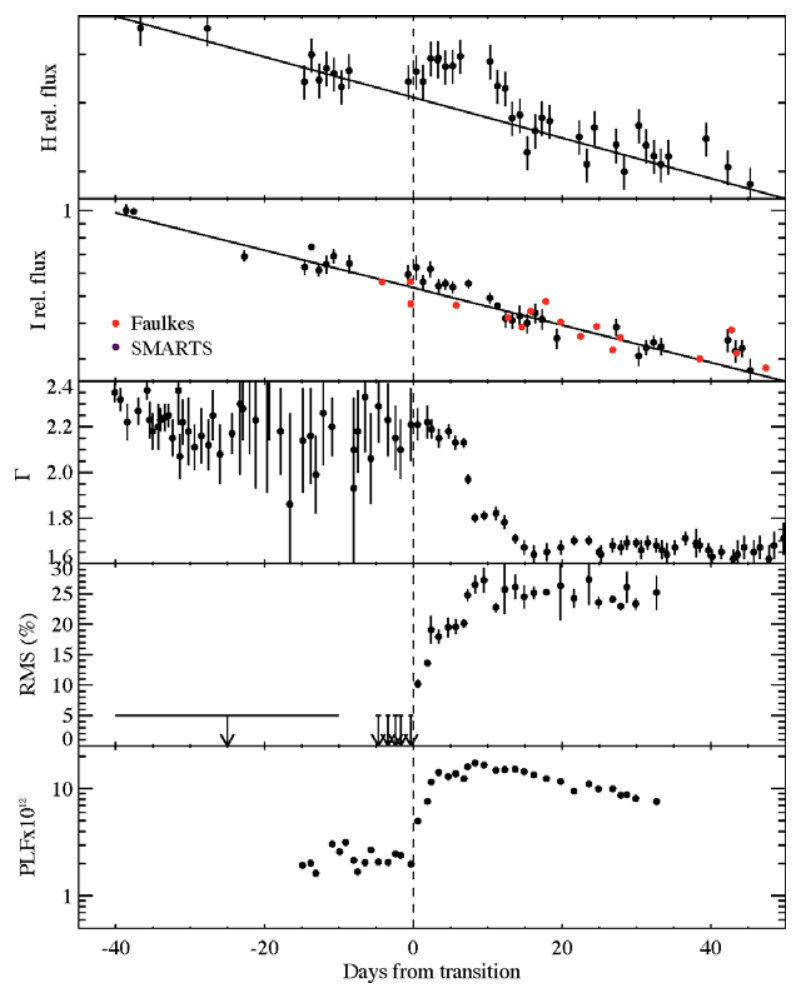

Figure 1. Evolution of NIR (H band) and optical (I band) relative flux with respect to X-ray spectral (spectral index, $\Gamma$ and power-law flux, PLF) and timing (RMS) evolution. Time 0 is chosen at the beginning of fast timing transition. 


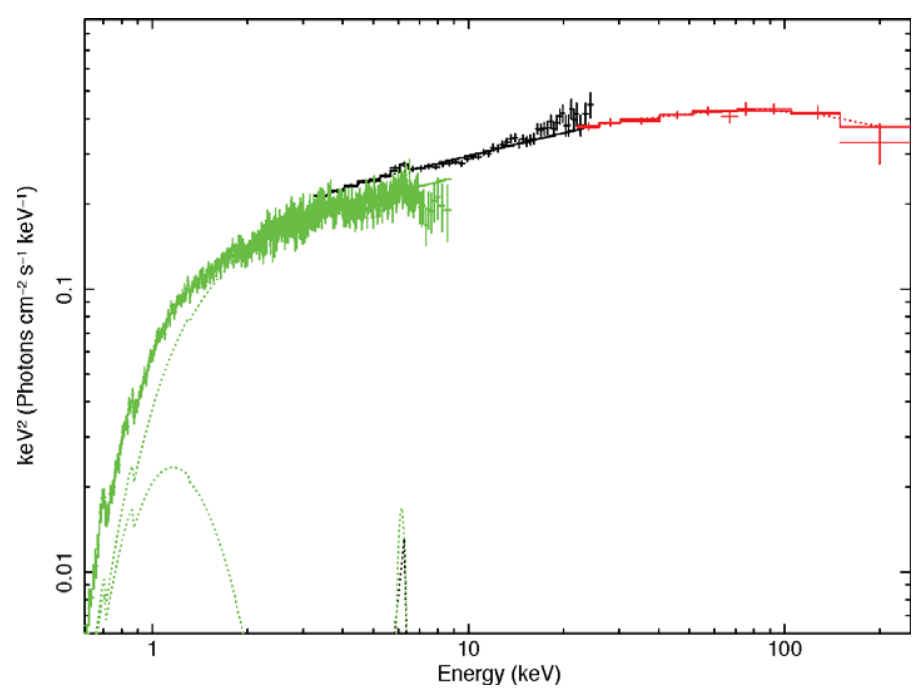

Figure 2. ISGRI, XRT and PCA combined spectrum.

power-law and a smeared edge. If necessary we added a Gauss to represent the iron line. We also created power spectra in 3-25 keV band. On MJD 55282.6 we observed timing features in the power density spectrum (PDS) with $10 \%$ rms variability. For observations before this date, the source was in the soft state with no significant timing features in the PDS. In time scale of 2 days, stronger broad band noise filled the PDS and the rms amplitude of variability increased to $20 \%$ (see Fig. 1).

In addition we extracted $\mathrm{H}$ and $\mathrm{I}$ band differential magnitudes using data from the SMARTS CTIO Telescope. The $\mathrm{H}$ band observations were fitted with an exponential decay to bring out the brightening in flux. This kind of brightening during outburst decays are common in GBHTs and are associated with optically thin synchrotron radiation from compact jets (Buxton, \& Bailyn, 2004, Kalemci, et al. 2005, Russell, et al. 2006, Coriat, et al. 2009) but other possibilities exist (thermal emission from disk, irradiation of secondary star Homan, et al. 2005). This is the first time that we observe an increase in NIR flux coinciding with timing transition, well before the hardening of spectrum. It is interesting to note that the optical data do not show a strong (if any) increase in flux. We are investigating the possibility that this increase is due to irradiation, rather than synchrotron, however, lack of strong brightening in optical is in favor of jet interpretation (see Fig. 1, top two panels, which also includes data from Faulkes Observatory, acknowledgment Dave Russell).

Finally we also investigated whether breaks disappear in the hard X-ray spectrum after the jet turns as observed in some other sources (Kalemci, et al. 2005, Caballero-Garcia, et al. 2007), by modeling the INTEGRAL ISGRI spectrum together with SWIFT XRT and $R X T E$ PCA. The $\sim 300 \mathrm{ks} I N T E G R A L$ observation was taken around 20 days after the transition. Fig. 2 shows the spectrum and the fit model that consists of absorption, disk blackbody, a power-law with a cutoff and a gaussian. The spectrum clearly requires a break, with folding energy of $\sim 270 \mathrm{keV}$. If these folding energies are typical for GBHTs after jet turn on, this may explain why HEXTE would miss such breaks for typical short observing observations with $R X T E$ for other sources. 


\section{Inner disk radius and jets}

One of the factors that may be relevant for jet formation is the optically thick disk inner radius. It has already been shown that disk flux should be less than a few percent of the total flux in the $3-25 \mathrm{keV}$ band to be able to observe compact jets during outburst decays (Kalemci, et al. 2006a). It has been suggested that geometrically thick disks are prerequisite for launching jets (Meier, Koide, \& Uchida, 2001), and it is been shown that a coronal mechanism is more likely to transport magnetic field inwards than the optically thick disk (Beckwith, Hawley, \& Krolik, 2009). A simple explanation would be a receding accretion disk, and a corona filling the gap before launching the jet.

If this is the case, then we should see evidence of a recessing disk in the hard state before the jet turns on. On the other hand, several work concentrating on the iron line reflection features, and diskblackbody normalization provide result consistent with disk staying close to the innermost stable orbit (Reis, et al. 2010, and references therein, Tomsick, et al. 2008, but also see Done, \& Diaz-Trigo, 2010). In most of these cases, there is evidence of a compact jet. Therefore, the jets can form when the disk is close to the last stable orbit.

We also investigate the relation between jets and inner disk radius further by the analysis of iron line profile at very low fluxes in the hard state. We detected a narrow line at $0.14 \%$ Eddington Luminosity for GX 339-4 with SUZAKU, and placed the inner disk radius $105 R_{g}$ at $18^{\circ}$ inclination. The disk recede, while the jet is still active. We conclude that the development of the corona plays an important role on formation of jets, and low disk fluxes are required for this, however the inner disk radius does not play a role on the formation of jets.

\section{References}

Beckwith, K., Hawley, J. F \& Krolik, J. H. 2009, ApJ 707, 428

Brocksopp, C. et al. 2009, ATEL 2278.

Buxton, M. M. \& Bailyn, C. D. et al. 2004, ApJ 615, 880

Buxton, M. M. et al. 2009, ATEL 2549.

Caballero Garcia, M. D. et al. 2007, ApJ 669, 534

Curran, P. A. et al. 2010, MNRAS, accepted for publication

Coriat, M. et al. 2009, MNRAS, 400, 123

Done, C. \& Diaz-Trigo, M. 2010, MNRAS, 407, 2287

Homan, J. et al. 2005, ApJ, 624, 295

Kalemci, E. et al. 2005, ApJ, 622, 508.

Kalemci, E. et al. 2006a, in Proceedings of the 6th Microquasar Workshop, 12

Kalemci, E. et al. 2006b, ApJ, 639, 340.

Nakahira, N. et al. 2010, PASJ, accepted for publication

Meier, D. L. Koide, S., \& Uchida, Y. 2001, Science 291, 84

Markwardt, C. B. et al. 2009, ATEL 2258.

Munoz-Darias, T. et al. 2010, MNRAS, 404, L94

Reis, R. C. et al. 2010, MNRAS, 402, 836

Russell, D. M. et al. 2006, MNRAS, 371, 1334

Russell, D. M. \& Fender, R. et al. 2010a, Invited chapter for the edited book "Black Holes and Galaxy Formation", Nova Science Publishers, Inc., at press

Shaposhnikov, N. et al. 2010, ApJ, accepted for publication

Tomsick, J. A. et al. 2008, ApJ, 680, 593

Tomsick, J. A. et al. 2009, ApJL, 707, L87

Yang, J. et al. 2010, MNRAS, accepted for publication 